\title{
The Cylindrical Wave Approach for the Electromagnetic Scattering by Targets behind a Wall
}

\author{
Cristina Ponti $1,2, *(1)$ and Giuseppe Schettini ${ }^{1,2}$ \\ 1 Department of Engineering, “Roma Tre” University, via Vito Volterra 62, 00146 Rome, Italy; \\ giuseppe.schettini@uniroma3.it \\ 2 National Interuniversity Consortium for Telecommunications, Roma Tre University, 00146 Roma, Italy \\ * Correspondence: cristina.ponti@uniroma3.it; Tel.: +39-0657337358
}

Received: 30 September 2019; Accepted: 28 October 2019; Published: 1 November 2019

\begin{abstract}
An overview of the cylindrical wave approach in the modeling of through-wall radar problems with targets hidden behind a dielectric wall is reported. The cylindrical wave approach is a technique for the solution of the two-dimensional scattering by buried circular cross-section cylinders in a semi-analytical way, through expansion of the scattered fields into cylindrical waves. In a through-wall radar application, the scattering environment is made by a dielectric layer between two semi-infinite half-spaces filled by air. For this layout, two possible implementations of the cylindrical wave approach have been developed in the case of plane-wave excitation. The first was an iterative scheme with multiple-reflection scattered fields, and the second was a fast and non-iterative solution, through suitable basis functions (i.e., reflected and transmitted cylindrical waves). Such waves take into account all the interactions of the source field with the interfaces bounding the dielectric layers and the targets. The non-iterative approach was also extended for excitation from the radiated field by a line source. A final system was derived for the computation of the scattered field by PEC or dielectric targets. Numerical results show the potentialities of the cylindrical wave approach in the modeling of through-wall radar, in particular in the evaluation of the scattered fields by human targets in a building's interior, modeled with a two-dimensional approach.
\end{abstract}

Keywords: electromagnetic scattering; buried objects; through-wall radar

\section{Introduction}

Through-the-wall radar is a technique to localize targets hidden by walls in a building's interior [13]. Important operations are in the field of security, military applications, such as counterterrorism or civil investigations, such as rescue, disaster, and fire succor. This radar technique typically makes use of ultrawideband waveforms to achieve high resolutions through very narrow pulses. However, the highest frequencies of the spectrum have small penetration through typical walls, and part of the field undergoes multiple reflections inside the wall. An accurate electromagnetic modeling of the scattering occurring in through-wall radar applications is fundamental to enhance the understanding of the physical phenomena, also serving as a benchmark of the imaging algorithms. In the literature, the electromagnetic modeling of through-wall radar is mainly done with the finite-difference time-domain (FDTD) method, as in [4], where an FDTD implemented for ground penetrating radar was employed [5]. A general FDTD approach was also employed in [6]. A specific FDTD approach for the through-wall analysis in time domain, called AFDTD, was developed in [7]. The AFDTD is based on the classical Yee algorithm, where the volume of analysis is divided in a grid of cubic cells, and its primary application is to radar target signature analysis [1]. Due to the large investigation domains dealt with in through-wall environments, hybrid techniques are also proposed, as in [8,9], where FDTD is combined with high-frequency techniques, such as ray tracing or uniform theory of diffraction. 
Another hybrid approach was presented in [10], where integral equations were solved with physical optics. An asymptotic technique was employed in [11]. A frequency-domain finite element method was also proposed in [12]. All the techniques were developed on a numerical basis and approximated to overcome limitations of long computer times and strong memory requirement needed in large simulation environments as is typical with most through-wall applications.

In this paper, an approach for the modeling of the scattering by targets behind a wall on an analytical basis is reviewed. The technique, called cylindrical wave approach (CWA), has been developed to analytically solve the two-dimensional scattering by buried or hidden targets having a circular cross-section [13-18], for applications in the geophysical analysis and remote sensing of subsoil, in the microwave frequency range, and as a diagnostic at optical frequencies. Expansions into cylindrical waves are used to express the fields scattered by the targets. The interactions of the scattered fields with one or more flat interfaces, bounding a semi-infinite medium or a layered one, are solved using the concept of plane wave spectrum of a cylindrical wave. By expressing a cylindrical wave through its plane wave spectrum, reflected and transmitted cylindrical waves can be suitably derived to express all the contributions to the scattered field. In $[19,20]$, the CWA formulation has been extended to deal with problems of scattering by cylinders below a dielectric layer, in case of a planewave source. In this layout, three background media are considered (i.e., an air-filled half-space, a dielectric layer, and a final half-space embedding the targets). Applications are both in the remote sensing of buried targets in the subsoil, as well as in the simulation of the through-wall environments when the final half-space is filled with air. In [19], the CWA was presented with an iterative scheme where all the multiple reflections of the scattered fields occur inside the dielectric layers where described, through a set of multiple reflection fields. The method was implemented in a faster approach in [20], where only two sets of scattered fields were introduced that, through the definitions of the reflection and transmission coefficients relevant to a dielectric layer [21], included all the multiple reflections in the expressions of the scattered fields. Agreement between the two methods was also presented in [20]. Both methods have the advantage of providing an accurate analytical tool with fast computer times in large through-wall environments. Moreover, with the non-iterative scheme in [20], execution times were considerably reduced compared to those in [19], as proven in an extensive comparison presented in [20]. This comparison showed the dependence of the two methods on the simulation parameters, including as radius of the cylinder, the thickness and permittivity of the wall, and the number of cylinders. In this paper, the non-iterative approach developed in [20] was recalled and extended to excitation from a line source. The radiated field was expanded through a zeroth-order Hankel function, and its reflection and transmission through the interfaces were dealt with by applying the same spectral approach employed for the scattered fields. As for the numerical implementation, potentialities of the CWA for the modeling in through-wall environments are presented.

This paper is organized as follows: In Section 2, the theoretical approach, applied to a line-source excitation, is presented. In Section 3, numerical results of through-wall simulation are reported.

\section{Theoretical Approach}

The geometry of the problem is sketched in Figure 1. In medium 0 (air, $\varepsilon_{\mathrm{r} 0}=1$ ), the source of the scattering problem, a line source with center in $\left(x_{\mathrm{L}}, z_{\mathrm{L}}\right)$, is present. $N$ cylinders, perfectly conducting or dielectric, are placed in an air-filled medium with relative permittivity $\varepsilon_{\mathrm{r} 2}=1$ (medium 2), below a dielectric layer of thickness $L$ and relative permittivity $\varepsilon_{\mathrm{r} 1}$ (medium 1$)$. The model is two-dimensional, assuming the length of the targets is much longer than the size of their cross-section. 


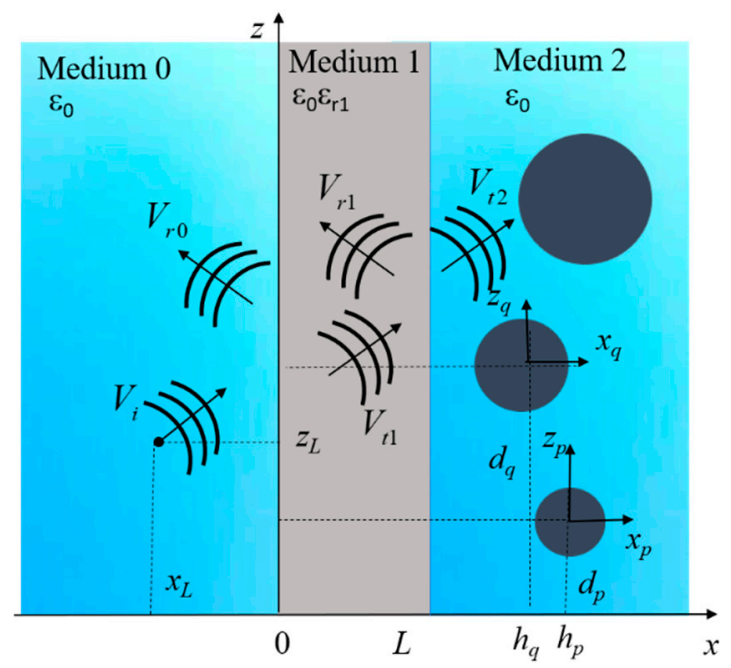

Figure 1. Geometry of the scattering problem. The incident field and the relevant reflected and transmitted fields in the absence of the targets are also highlighted.

Normalized coordinates $(\xi, \zeta)$ are employed, $\xi=\mathrm{k}_{0} \mathrm{x}$ and $\zeta=\mathrm{k}_{0} \mathrm{z}$, with $\mathrm{k}_{0}$ being the vacuum wavenumber. $N$ reference frames $\left(\xi_{p}, \zeta_{p}\right)$ centered on the axis of the $p$-th are also introduced, and the $p$-th cylinder has radius $a_{\mathrm{q}}$ and center in $\left(h_{\mathrm{q}}, d_{\mathrm{q}}\right)$, the normalized radius being $\alpha_{\mathrm{p}}=\mathrm{k}_{0} a_{\mathrm{p}}$, and the normalized center $\left(\chi_{\mathrm{p}}=\mathrm{k}_{0} h_{\mathrm{p}}, \eta_{\mathrm{p}}=\mathrm{k}_{0} d_{\mathrm{p}}\right)$, with $p=1, \ldots, N$. Normalized thickness of the interface is $\Lambda$ $=\mathrm{k}_{0} L$. The normalized coordinates of the center of the line source are given by $\left(\chi_{\mathrm{L}}, \eta_{\mathrm{L}}\right)$.

A scalar function $V(\xi, \zeta)$ is used to represent the component of the electromagnetic field parallel to the cylinders' axis, depending on the polarization state. It is $V=E_{\mathrm{y}}$ in the TM or E polarization, and $V$ $=H_{\mathrm{y}}$ in the TE or H polarization.

Here, the non-iterative approach is presented in the case of excitation with a line source, defined through an amplitude $V_{0}$ and proportional to the zeroth-order first-kind Hankel function $H_{0}^{(1)}(\cdot)$ [22]:

$$
V_{\mathrm{i}}(\xi, \zeta)=-V_{0} H_{0}^{(1)}\left[\sqrt{\left(\xi-\chi_{L}\right)^{2}+\left(\zeta-\eta_{L}\right)^{2}}\right] .
$$

An alternative representation is now introduced for the cylindrical wave in Equation (1). With the position $C W_{0}(\xi, \zeta)=H_{0}^{(1)}(\xi, \zeta), C W_{0}$ is a cylindrical wave of zeroth-order that can be defined through a spectral representation:

$$
C W_{0}(\xi, \zeta)=\frac{1}{2 \pi} \int_{-\infty}^{+\infty} F_{0}\left(\xi, \mathrm{n}_{\|}\right) e^{i n_{\|} \zeta} \mathrm{dn} \|,
$$

where the plane wave spectrum $F_{0}\left(\xi, \mathrm{n}_{\|}\right)$is given by:

$$
F_{0}\left(\xi, \mathrm{n}_{\|}\right)=\frac{2 e^{i \xi \sqrt{1-\mathrm{n}_{\|}^{2}}}}{\sqrt{1-\mathrm{n}_{\|}^{2}}}
$$

By evaluating the reflected or transmitted plane wave on each plane wave of the spectrum (Equation (2)), the fields originated by reflection and transmission of the incident field $V_{\mathrm{i}}$-in the absence of the cylinders, as highlighted in Figure 1—can be derived. The amplitudes of the plane waves are derived through reflection and transmission coefficients, with definition dealing with the interaction of a plane wave with a dielectric slab [21], which includes all the multiple reflections by the flat boundaries. As for the propagation terms, they are derived from the parallel and orthogonal components $n_{\|}$and $n_{\perp}$, respectively, of the normalized wavevector $\mathbf{n}=n_{\perp} \hat{\xi}+n_{\|} \hat{\zeta}=\mathbf{k} / k_{0}$ through the 
Snell law, being $n_{\perp}=\cos \varphi$ and $n_{\|}=\sin \varphi$. In particular, the field $V_{\mathrm{t} 2}$ transmitted in the final medium can be expressed through a transmitted cylindrical function of zeroth-order:

$$
T W_{0}\left(\xi, \zeta ;-\chi_{L} ; \Lambda\right)=\frac{1}{2 \pi} \int_{-\infty}^{+\infty} \mathrm{T}_{02}\left(\mathrm{n}_{\|}\right) F_{0}\left(-\chi_{L}, \mathrm{n}_{\|}\right) e^{i \mathrm{n}_{2} \sqrt{1-\left(\mathrm{n}_{\|} / \mathrm{n}_{2}\right)^{2}}(\xi-\Lambda)} e^{i \mathrm{n}_{\|}\left(\zeta-\eta_{L}\right)} \mathrm{dn}_{\|},
$$

where $n_{\perp}=\sqrt{1-\left(\mathrm{n}_{\|} / \mathrm{n}_{2}\right)^{2}}$ and $n_{\|}$are the orthogonal and parallel components, respectively, of a generic plane wave transmitted in medium 2.

The final expression of the field $V_{\mathrm{t} 2}$ is:

$$
V_{\mathrm{t} 2}(\xi, \zeta)=-V_{0} T W_{0}(\xi, \zeta)
$$

The other field contributions $\left(V_{\mathrm{r} 0}, V_{\mathrm{r} 1}\right.$, and $\left.V_{\mathrm{t} 1}\right)$, relevant to reflection and transmission of the incident field by the layer can be derived in a similar manner through suitable cylindrical functions, as in Equation (4).

As the field $V_{\mathrm{t} 2}$ impinges on the targets in medium 2, the following scattered field contributions are excited, as depicted in Figure 2a:

- $\quad V_{s}(\xi, \zeta)$ : fields scattered by the cylinders in medium 2;

- $V_{s r}^{2}(\xi, \zeta)$ : scattered-reflected field in medium 2 , by the interface in $\zeta=\Lambda$;

- $V_{s t}^{1}(\xi, \zeta)$ : scattered-transmitted field in medium 1 , by the interface in $\zeta=\Lambda$;

- $V_{s r}^{1}(\xi, \zeta)$ : scattered-reflected field in medium 1 , by the interface in $\zeta=0$;

- $V_{s t}^{1}(\xi, \zeta)$ : scattered-reflected field in medium 0 , by the interface in $\zeta=0$.

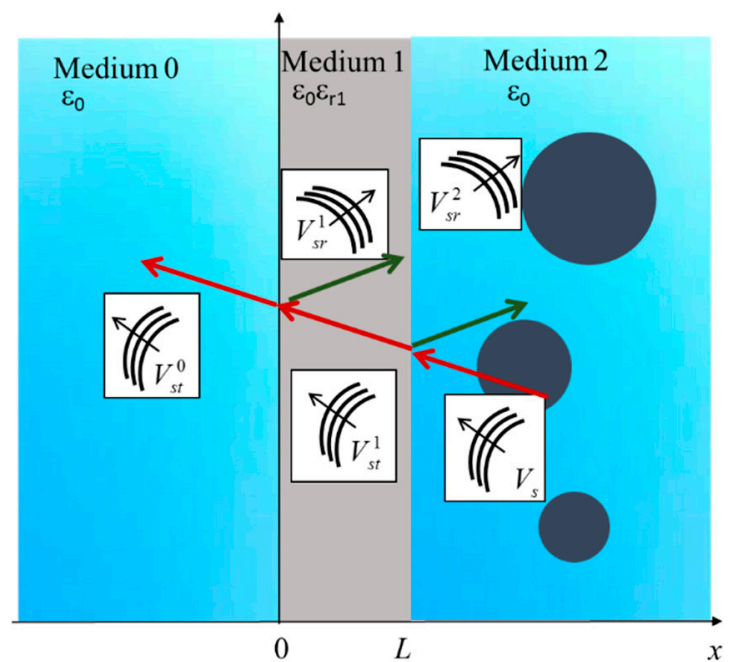

(a)

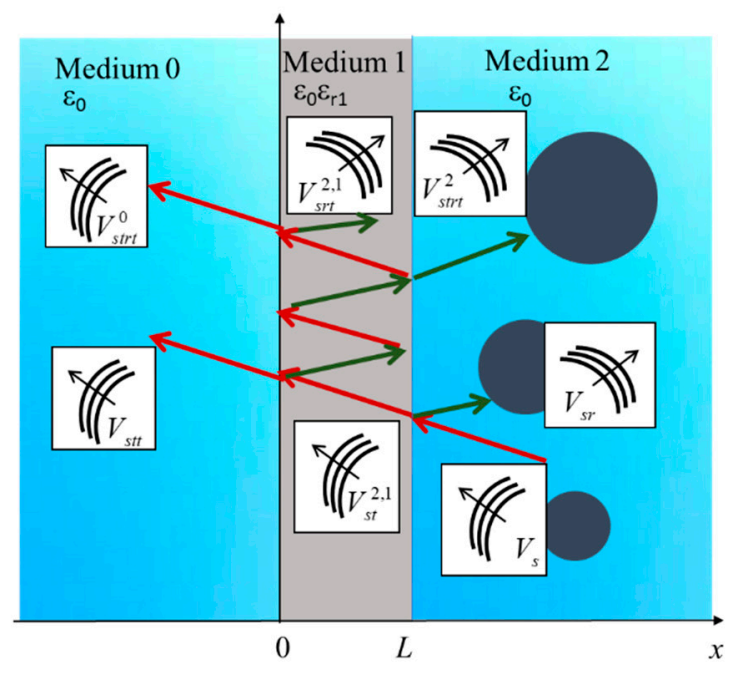

(b)

Figure 2. Decomposition of the scattered fields in the scattering by targets in a through-wall environment: (a) non-iterative approach [19] and (b) iterative approach [20].

With the field decomposition presented above, all the multiple interactions experienced by the scattered fields inside the layer comprise two sets of waves. The first is a left-propagating wave, which from medium 2 is scattered into medium 0 and decomposed into the fields $V_{s}, V_{s t}^{1}$ and $V_{s t}^{0}$. The second is a right-propagating wave, which from medium 0 is scattered back in medium 2, through the fields $V_{s r}^{2}$ and $V_{s r}^{1}$. This compact decomposition, presented in detail in [20], leads to a faster approach to the solution of the scattering problem, compared to the one of Figure 2b [19]. In the latter, all 
the possible interactions of the scattered fields inside the boundary are taken into account, through multiple reflection fields $V_{s t r t}^{0}(\xi, \zeta), V_{s r t}^{2,1}(\xi, \zeta)$, and $V_{s t r t}^{2}(\xi, \zeta)$. Such fields are given by infinite and convergent series, which are truncated to a finite number of terms in the numerical implementation depending on the desired accuracy.

In both approaches in $[19,20]$, the scattered field $V_{s}$ in medium 2 by the cylinders can be defined through an expansion into cylindrical functions of $m$-th order:

$$
C W_{m}\left(n_{1} \xi_{q}, n_{1} \zeta_{q}\right)=H_{m}^{(1)}\left(n_{1} \rho_{q}\right) e^{i m \theta_{q}}
$$

where $H_{m}^{(1)}$ is the first-kind Hankel function of order $m$ and $e^{i m \theta_{q}}$ is an angular factor. Employing the basis functions in Equation (6), the scattered field by $N$ cylinders is given by:

$$
V_{\mathrm{s}}\left(\xi_{\mathrm{q}}, \zeta_{\mathrm{q}}\right)=V_{0} \sum_{\ell=-\infty}^{\infty} J_{\ell}\left(\mathrm{n}_{2} \rho_{\mathrm{p}}\right) e^{i \ell \theta_{\mathrm{p}}} \sum_{\mathrm{q}=1}^{\mathrm{N}} \sum_{m=-\infty}^{+\infty} i^{\mathrm{m}} c_{\mathrm{qm}}\left[C W_{\mathrm{m}-\ell}\left(\mathrm{n}_{2} \xi_{\mathrm{qp}}, \mathrm{n}_{2} \zeta_{\mathrm{qp}}\right)\left(1-\delta_{\mathrm{qp}}\right)+\frac{H_{\ell}^{(1)}\left(\mathrm{n}_{2} \rho_{\mathrm{p}}\right)}{J_{\ell}\left(\mathrm{n}_{2} \rho_{\mathrm{p}}\right)} \delta_{\mathrm{qp}} \delta_{\ell \mathrm{m}}\right]
$$

where $c_{\mathrm{qm}}$ are unknown expansion coefficients, and $\delta_{\mathrm{qp}}$ and $\delta_{\ell m}$ are the Kronecker symbols.

The definition in Equation (7), obtained by applying the addition theorem of Hankel functions and the plane wave expansion of a cylindrical wave, returns an expression of the scattered fields in polar coordinates centered on the axis of the $q$-th cylinder [13]. A representation in a polar reference frame is indeed adopted for the fields propagating in medium 2, being more suitable to apply boundary conditions on cylinders' surfaces, as is done in the following explanation.

The scattered field contributions in both approaches $[19,20]$ are derived through suitable cylindrical functions from the cylindrical functions of order $m$ in Equation (6), expressed through its plane wave spectrum [13]:

$$
C W_{\mathrm{m}}(\xi, \zeta)=\frac{1}{2 \pi} \int_{-\infty}^{+\infty} F_{\mathrm{m}}\left(\xi, \mathrm{n}_{\|}\right) e^{i \mathrm{n}_{\|} \zeta} \mathrm{dn} \|
$$

being:

$$
F_{\mathrm{m}}\left(\xi, \mathrm{n}_{\|}\right)=\frac{2 e^{i|\xi|} \sqrt{1-\mathrm{n}_{\|}^{2}}}{\sqrt{1-\mathrm{n}_{\|}^{2}}}\left\{\begin{array}{cc}
e^{-i \operatorname{marccosn}_{\|}}, & \xi \geq 0 \\
e^{i \operatorname{marccos} \|_{\|}}, & \xi \leq 0
\end{array}\right.
$$

with $n_{\|}$and $n_{\perp}$ the parallel and orthogonal component, respectively, of the normalized wavevector $\mathbf{n}=n_{\perp} \hat{\xi}+n_{\|} \hat{\zeta}=\mathbf{k} / k_{0}$. The expressions in Equations (8) and (9) generalize the ones in Equations (2) and (3), respectively, to a cylindrical wave of $m$-th order. Therefore, with an approach analogous to the one employed to derive the transmitted function in Equation (4) from the incident field $V_{\mathrm{i}}$, it is possible to derive the cylindrical waves to be used as basis functions of the reflected and transmitted scattered fields. The further scattering contributions are presented for the decomposition of Figure 2a. A scattered reflected field is excited by the interaction of the field $V_{\mathrm{s}}$ with the flat boundary in $\xi=\Lambda$ of separation between medium 1 and medium 2. It is expressed through an expansion into reflected cylindrical waves in medium 2:

$$
R W_{m}\left(n_{2} \xi, n_{2} \zeta\right)=\frac{1}{2 \pi} \int_{-\infty}^{+\infty} \Gamma_{21}\left(n_{\|}\right) F_{\mathrm{m}}\left[n_{2}(\xi-\Lambda), n_{\|}\right] e^{i n_{1} n_{\|} \zeta} d n_{\|}
$$

In Equation (10), the reflection coefficient $\Gamma_{21}\left(n_{\|}\right)$at the medium 1/medium 2 boundary is the one for a dielectric slab [21]. The cylindrical waves in Equation (10) are employed as basis functions of the scattered reflected field in medium 2 :

$$
V_{\mathrm{sr}}\left(\xi_{\mathrm{q}}, \zeta_{\mathrm{q}}\right)=V_{0} \sum_{\ell=-\infty}^{+\infty} J_{\ell}\left(\mathrm{n}_{2} \rho_{\mathrm{p}}\right) e^{i \ell \theta_{\mathrm{p}}} \sum_{\mathrm{q}=1}^{\mathrm{N}} \sum_{\mathrm{m}=-\infty}^{+\infty} c_{\mathrm{qm}} R W_{\mathrm{m}+\ell}\left[-\mathrm{n}_{2}\left(\Lambda-\chi_{\mathrm{q}}+\chi_{\mathrm{p}}\right), \mathrm{n}_{2}\left(\eta_{\mathrm{q}}-\eta_{\mathrm{p}}\right)\right]
$$


As explained above for the scattered field in Equation (7), the definition of the scattered-reflected field in Equation (11) is expressed in a polar reference frame centered on the $q$-th cylinder.

As for the field contributions in medium 1, they are expressed through the transmitted cylindrical waves in medium 1 :

$$
T W_{m}^{1}\left(\xi, \zeta ; \chi_{q} ; \Lambda\right)=\frac{1}{2 \pi} \int_{-\infty}^{+\infty} T_{21}\left(n_{\|}\right) F_{\mathrm{m}}\left[-n_{2}\left(\chi_{q}-\Lambda\right), n_{\|}\right] e^{i n_{1} \sqrt{1-\left(n_{2} n_{\|} / n_{1}\right)^{2}}(\xi-\Lambda)} e^{i n_{2} n_{\|}\left(\zeta-\eta_{q}\right)} d n_{\|}
$$

and the reflected cylindrical waves in medium 1:

$$
R W_{m}^{1}\left(\xi, \zeta ; \chi_{q} ; \Lambda\right)=\frac{1}{2 \pi} \int_{-\infty}^{+\infty} T_{21}\left(n_{\|}\right) \Gamma_{10}\left(n_{\|}\right) F_{\mathrm{m}}\left[-n_{2}\left(\chi_{q}-\Lambda\right), n_{\|}\right] e^{i n_{1} \sqrt{1-\left(n_{2} n_{\|} / n_{1}\right)^{2}}(\xi+\Lambda)} e^{i n_{2} n_{\|}\left(\zeta-\eta_{q}\right)} d n_{\|}
$$

Equations (12) and (13) are used as basis functions of the scattered-transmitted and scattered-reflected field in medium 1, respectively, represented through:

$$
V_{s r}^{1}(\xi, \zeta)=V_{0} \sum_{\mathrm{q}=1}^{\mathrm{N}} \sum_{\mathrm{m}=-\infty}^{+\infty} c_{\mathrm{qm}} R W_{m}^{1}\left(\xi, \zeta ; \chi_{q} ; \Lambda\right)
$$

and:

$$
V_{s t}^{1}(\xi, \zeta)=V_{0} \sum_{\mathrm{q}=1}^{\mathrm{N}} \sum_{\mathrm{m}=-\infty}^{+\infty} c_{\mathrm{qm}} T W_{m}^{1}\left(\xi, \zeta ; \chi_{q} ; \Lambda\right)
$$

Finally, transmitted cylindrical waves in medium 0 are introduced:

$$
T W_{m}^{0}\left(\xi, \zeta ; \chi_{q}\right)=\frac{1}{2 \pi} \int_{-\infty}^{+\infty} T_{10}\left(n_{\|}\right) T_{21}\left(n_{\|}\right) F_{\mathrm{m}}\left[-n_{2}\left(\chi_{q}-\Lambda\right), n_{\|}\right] e^{i \sqrt{1-\left(n_{2} n_{\|}\right)^{2}} \xi} e^{i n_{2} n_{\|}\left(\zeta-\eta_{q}\right)} d n_{\|}
$$

and they are employed as basis functions of the scattered-transmitted field in air:

$$
V_{s t}^{0}(\xi, \zeta)=V_{0} \sum_{\mathrm{q}=1}^{\mathrm{N}} \sum_{\mathrm{m}=-\infty}^{+\infty} c_{\mathrm{qm}} T W_{m}^{0}\left(\xi, \zeta ; \chi_{q}\right)
$$

In the case of dielectric targets of relative permittivity $\varepsilon_{\mathrm{rc}}$, a further field contribution must be included (i.e., the scattered field transmitted inside the cylinders). Its representation is given through an expansion in first-kind Bessel functions $J_{\ell}(\cdot)$ and a second set of unknown expansion coefficients $d_{p \ell}$ :

$$
V_{s c}(\xi, \zeta)=V_{0} \sum_{\mathrm{q}=1}^{\mathrm{N}} \sum_{\mathrm{m}=-\infty}^{+\infty} d_{p \ell} J_{\ell}\left(n_{c} \rho_{c}\right)
$$

For the determination of the expansion coefficients $c_{q m}$ in the scattered fields-Equations (7), (11), (14), (15), (17), and the coefficients $d_{p \ell}$ of the field in Equation (18) - the boundary conditions relevant to the tangential fields to the cylinders' surface are imposed. Therefore, only the field's components propagating in medium 2 are involved and are expressed in polar coordinates as already implemented in Equations (5), (7), (11), and (18). 
In TM polarization, boundary conditions are:

$$
V_{\mathrm{t} 2}+V_{\mathrm{s}}+\left.V_{s r}^{2}\right|_{\rho_{\mathrm{p}}=\alpha_{\mathrm{p}}}=\left.V_{s c}\right|_{\rho_{\mathrm{p}}=\alpha_{\mathrm{p}}}
$$

where in the TE polarization states it is:

$$
\left.\frac{\partial}{\partial \rho_{\mathrm{p}}}\left(V_{\mathrm{t} 2}+V_{\mathrm{s}}+V_{s r}^{2}\right)\right|_{\rho_{\mathrm{p}}=\alpha_{\mathrm{p}}}=\left.\frac{\partial}{\partial \rho_{\mathrm{p}}}\left(V_{\mathrm{sc}}\right)\right|_{\rho_{\mathrm{p}}=\alpha_{\mathrm{p}}}
$$

with $p=1, \ldots, N$. In the case of PEC targets, the second member of the conditions (Equations (19) and (20)) reduces to zero. By introducing in Equations (19) and (10) the definitions of the fields in Equations (5), (7), (11), and (18), a linear system is obtained after some manipulation for the expansions coefficients $c_{q m}$ :

$$
\sum_{\mathrm{q}=1}^{\mathrm{N}} \sum_{\mathrm{m}=-\infty}^{+\infty} D_{\mathrm{qp}}^{\ell \mathrm{m}(1,2)} \mathrm{c}_{\mathrm{qm}}=M_{\mathrm{p}}^{\ell(1,2)}\left\{\begin{array}{c}
\mathrm{p}=1, \ldots, \mathrm{N} \\
\ell=0, \pm 1, \ldots, \pm \infty
\end{array}\right.
$$

In Equation (21), the superscripts $\gamma=1,2$ refer to the boundary conditions in Equations (19) and (20), respectively, and it is $D_{m \ell}^{q p}=G_{\ell}^{p(1)} A_{m \ell}^{q p(1)}-G_{\ell}^{p(1)} A_{m \ell}^{q p(2)}, M_{\ell}^{p}=B_{\ell}^{p(1)} G_{\ell}^{p(1)}-B_{\ell}^{p(2)} G_{\ell}^{p(2)}$ and:

$$
\begin{gathered}
\mathrm{A}_{\mathrm{qp}}^{\ell \mathrm{m}(\gamma)}=i^{-\ell} T_{\ell}^{(\gamma)}\left(\mathrm{n}_{1} \rho_{\mathrm{p}}\right)\left\{C W_{\mathrm{m}-\ell}\left(\mathrm{n}_{2} \xi_{\mathrm{qp}}, \mathrm{n}_{2} \zeta_{\mathrm{qp}}\right)\left(1-\delta_{\mathrm{qp}}\right)+\right. \\
\left.+R W_{\mathrm{m}+\ell}\left[-\mathrm{n}_{2}\left(\chi_{\mathrm{q}}+\chi_{\mathrm{p}}-2 \Lambda\right), \mathrm{n}_{2}\left(\eta_{\mathrm{q}}-\eta_{\mathrm{p}}\right)\right]+\frac{\delta_{\mathrm{qp}} \delta_{\ell \mathrm{m}}}{T_{\ell}^{(\gamma)}\left(\mathrm{n}_{2} \rho_{\mathrm{p}}\right)}\right\} \\
\mathrm{B}_{\mathrm{p}}^{\ell(\gamma)}=-T_{\ell}^{(\gamma)}\left(\mathrm{n}_{1} \rho_{\mathrm{p}}\right) T W_{0, \ell}\left(\chi_{\mathrm{p}}, \eta_{\mathrm{p}}-\eta_{\mathrm{L}}-\chi_{\mathrm{L}}\right)
\end{gathered}
$$

being $G_{\mathrm{p}}^{\ell(1)}=-J_{\ell}\left(n_{c p} \alpha_{p}\right) / H_{\ell}^{(1)}\left(n_{1} \alpha_{p}\right), \quad G_{\mathrm{p}}^{\ell(2)}=-g_{p} J_{\ell}\left(n_{c p} \alpha_{p}\right) / H_{\ell}^{(1) \prime}\left(n_{1} \alpha_{p}\right)$, and $T_{\ell}^{(1)}(x)=$ $-J_{\ell}(x) / H_{\ell}^{(1)}(x), T_{\ell}^{(2)}(x)=-J_{\ell}(x) / H_{\ell}^{(1) \prime}(x)$, where $g_{p}=n_{c p} / n_{1}$ or $n_{1} / n_{c p}$ for $\mathrm{TM}$ or $\mathrm{TE}$ polarization, respectively.

The coefficients $d_{p \ell}$ relevant to the scattered field transmitted inside the cylinders are found from:

$$
\begin{aligned}
& d_{p \ell}=i^{-\ell} \frac{J_{\ell}\left(n_{1} \alpha_{p}\right) H_{\ell}^{(1) \prime}\left(n_{1} \alpha_{p}\right)-J \gamma_{\ell}\left(n_{1} \alpha_{p}\right) H_{\ell}^{(1)}\left(n_{1} \alpha_{p}\right)}{n_{1} J_{\ell}\left(n_{1} \alpha_{p}\right) H_{\ell}^{(1) \prime}\left(n_{1} \alpha_{p}\right)-n_{c p} J_{\ell}\left(n_{1} \alpha_{p}\right) H_{\ell}^{(1)}\left(n_{1} \alpha_{p}\right)}
\end{aligned}
$$

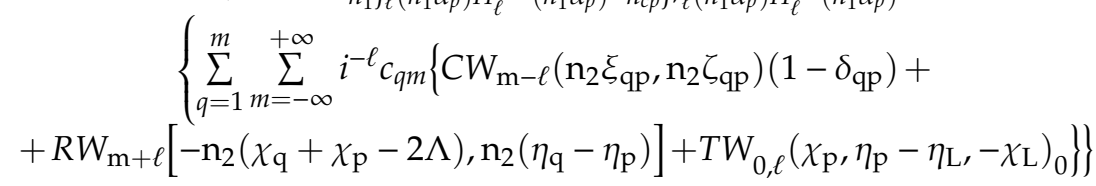

With PEC targets, the final system is the same as in Equation (21), with $\mathrm{D}_{\mathrm{qp}}^{\ell \mathrm{m}}(\gamma)$ equal to the matrix $A_{\mathrm{qp}}^{\ell \mathrm{m}(\gamma)}$ in Equation (22), and the term $M_{\mathrm{p}}^{\ell(\gamma)}$ corresponding to the definition $B_{\mathrm{p}}^{\ell(\gamma)}$ in Equation (23).

\section{Results}

The approach presented in Section 2 was solved numerically to model scattering applications of targets hidden behind a wall, as with the through-wall radar investigations. In the system (Equation (21)), the order $p$ and $\ell$ of the expansions into cylindrical waves were truncated to a finite number of terms $\left(M_{\mathrm{t}}\right)$. As a truncation rule, to give a compromise between accuracy and computational heaviness, a number of terms $M_{t}=\left\lfloor 3 n_{1} \alpha\right\rfloor$ [23] were employed in numerical computations, where $\alpha$ was the radius of the largest cylinder and $n_{1}$ the refraction index of medium 1 . Therefore, the size of the system (Equation (21)) was $M=N \times\left(2 M_{t}+1\right)$. In the modeling of human targets in the typical frequency range of through-wall radars, the truncation number $M_{t}=\left\lfloor 3 n_{1} \alpha\right\rfloor$ can assume high values. Possible values of $M_{t}$ are reported in the plots of Figure 3, for different sizes of parts of the human body, 
including knee (radius $a=6 \mathrm{~cm}$ ), head (radius $a=10 \mathrm{~cm}$ ), and thorax (radius $a=15 \mathrm{~cm}$ ), evaluated at 1 $\mathrm{GHz}$ (Figure 3a) and $2 \mathrm{GHz}$ (Figure 3b), with $n_{1}=2$. The truncation $M_{\mathrm{t}}$ in this implementation of the CWA for the through-wall modeling was larger than with the geophysical analysis, as in [18], where targets like pipes and subservices were detected at the maximum frequency of $1 \mathrm{GHz}$. Instead, in the through-wall investigations, the size of the targets was larger compared to the operational frequencies, (i.e., a larger $a / \lambda$ ratio was employed). However, the following results confirm that the results of the CWA were stable and convergent, although a higher number of terms in the expansion of the scattered field must be included.

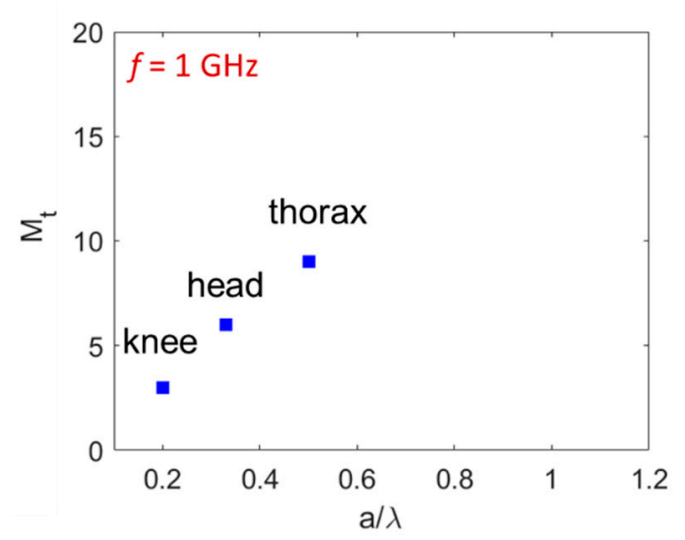

(a)

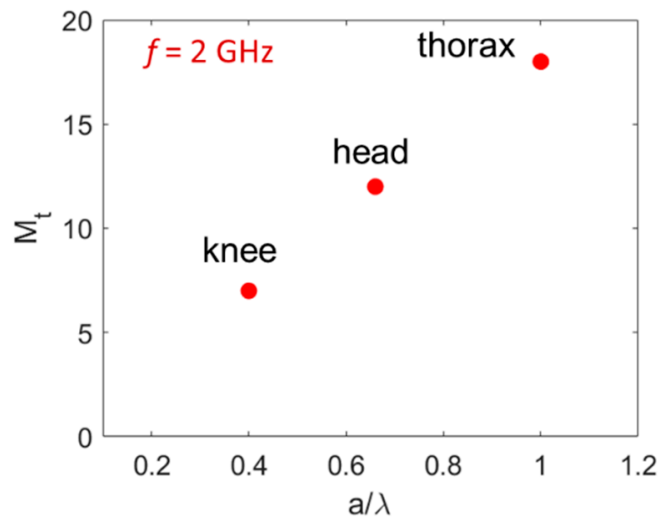

(b)

Figure 3. Truncation $M_{\mathrm{t}}$ on the field expansions based on the target size $2 a$ (knee $a=6 \mathrm{~cm}$, head $a=10$ $\mathrm{cm}$, thorax $a=15 \mathrm{~cm}$ ) at two different frequencies: (a) $1 \mathrm{GHz}$; and (b) $2 \mathrm{GHz}$.

Some results showing the potentialities of the method for the simulation of through-wall layouts are now illustrated. For target modeling, in the frequency range from 1 to $2 \mathrm{GHz}$, permittivity of biological tissues was high and can be represented either as a dielectric medium or as a PEC. In the dielectric modeling, a real part of the permittivity was $\varepsilon_{\mathrm{rc}}=50$, and an electrical conductivity $\sigma=1$ $\mathrm{S} / \mathrm{m}$ was assigned. In Figure 4, two-dimensional field maps are reported for the targets highlighted in Figure 3 (i.e., two knees (plots $(\mathrm{a}-\mathrm{c})$ ), head (plots $(\mathrm{d}-\mathrm{f})$ ), and thorax (plots $(\mathrm{g}-\mathrm{i})$ ), modeled as PEC targets under plane wave excitation (as in [20]) in TM polarization and normal incidence. As for the wall parameters, thickness was $L=20$ and relative permittivity was $\varepsilon_{\mathrm{r} 1}=4$. The total scattered field was evaluated in each medium, at the frequencies of $1 \mathrm{GHz}$ and $2 \mathrm{GHz}$. In Figure $3 \mathrm{~b}, \mathrm{c}$, effects of constructing interference between two interacting targets can be observed.

In the results of Figure 4, the use of a plane wave as incident field was equivalent to an excitation with a far-field source. The effect of excitation with a line source as in Equation (1) is shown in Figure 5. The target was modeled as a dielectric one, with radius $a=10 \mathrm{~cm}$, and center in $\left(h_{1}, d_{1}\right)=(0.6 \mathrm{~m}, 0.5 \mathrm{~m})$. The center of the line source was in $\left(\mathrm{x}_{\mathrm{L}}=-0.5 \mathrm{~m}, \mathrm{z}_{\mathrm{L}}\right)$, as the coordinate $\mathrm{z}_{\mathrm{L}}$ was moved from $-1 \mathrm{~m}$ to a position aligned with the cylinder axis, and the scattered field in medium 1 was collected along a line parallel to interface in $x=-50 \mathrm{~cm}$. The geometry of the problem is reported in Figure $5 \mathrm{a}$. The results in Figure $5 b$ show the reduction in amplitude for lateral positions of the source, for a wall with $L=20$ and $\varepsilon_{\mathrm{r} 1}=4$. This extension of the method to a line source excitation allows modeling of the multistatic acquisition employed in multifrequency approaches [24], as explored in recent algorithms for the imaging of targets in a through-wall environment [25]. 


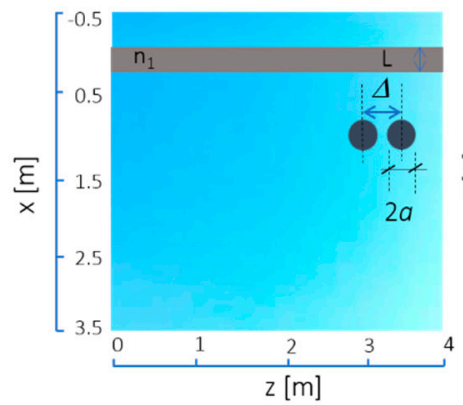

(a)

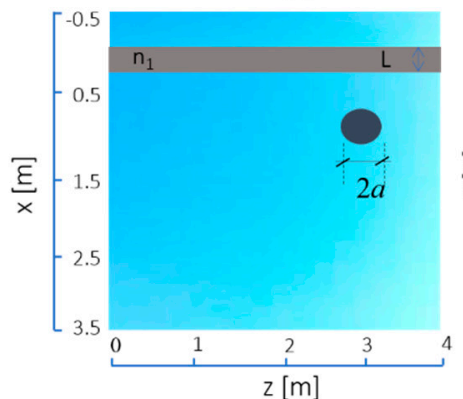

(d)

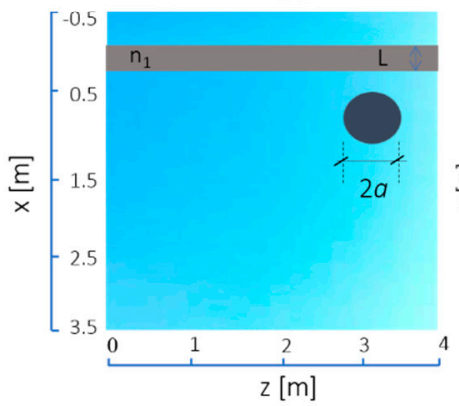

(g)

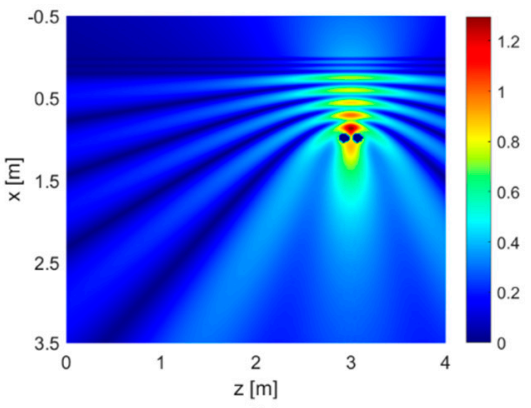

(b)

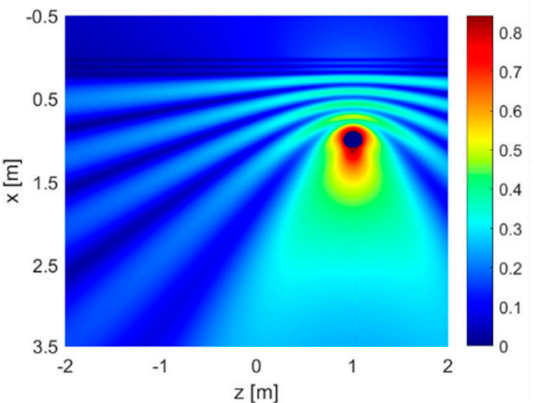

(e)

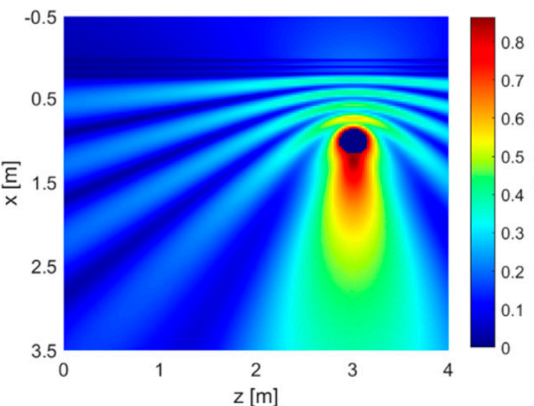

(h)

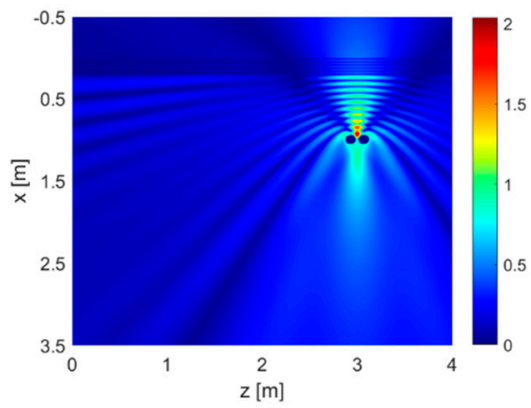

(c)

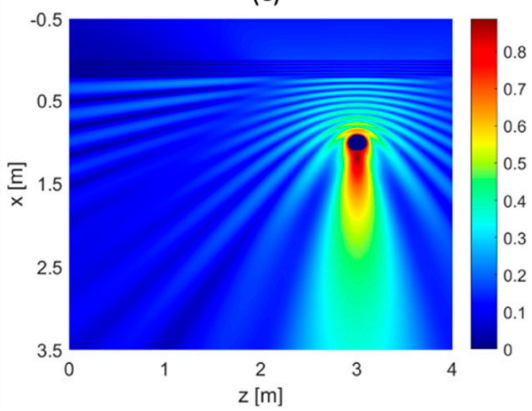

(f)

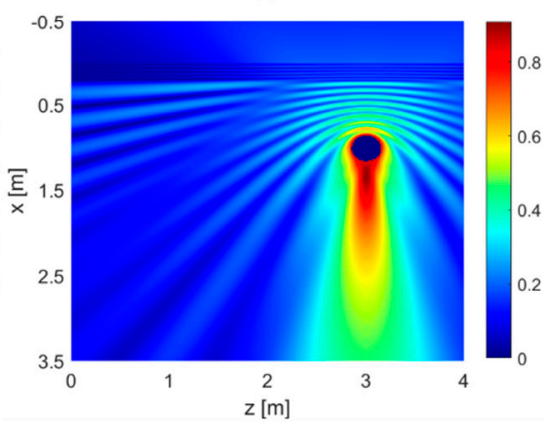

(i)

Figure 4. Two-dimensional field maps of the total electric field with targets modeled as PEC and plane wave excitation in TM polarization and normal incidence [20]: (a) knee $(a=6 \mathrm{~cm})$ at $1 \mathrm{GHz}(\mathbf{b})$ and 2 $\mathrm{GHz}(\mathbf{c}) ;(\mathbf{d})$ head $(a=10 \mathrm{~cm})$ at $1 \mathrm{GHz}(\mathbf{e})$ and $2 \mathrm{GHz}(\mathbf{f}) ;(\mathrm{g})$ thorax $(a=15 \mathrm{~cm})$ at $1 \mathrm{GHz}(\mathbf{h})$ and $2 \mathrm{GHz}$ (i). The dielectric layer has thickness $L=20 \mathrm{~cm}$ and $\varepsilon_{\mathrm{r} 1}=4$.

The far-field radar cross section (RCS) [17] as a function of the scattering $\theta$ is reported in Figure 6, evaluated in the presence of a wall $\left(L=20 \mathrm{~cm}, \varepsilon_{\mathrm{r} 1}=4\right)$, for a dielectric cylinder with permittivity of a biological medium $\left(\varepsilon_{\mathrm{rc}}=50, \sigma=1 \mathrm{~S} / \mathrm{m}\right)$ in Figure 6 , and for a low permittivity target modeling a wood $\operatorname{rod}\left(\varepsilon_{\mathrm{rc}}=2.5, \sigma=0.004 \mathrm{~S} / \mathrm{m}\right)$, at the frequency of $1 \mathrm{GHz}$, in Figure $6 \mathrm{~b}$. The targets have a fixed center in $(0,0.5 \mathrm{~cm})$, and two different radii of $7.5 \mathrm{~cm}$ and $15 \mathrm{~cm}$ are simulated. The source is given by a plane wave field. Comparison between the two dielectric targets showed a consistent reduction in the radar cross-section in the low-permittivity case which was more pronounced for the largest radius. The behavior of the radar cross section can also be estimated in the backscattering direction $\theta$ $=180^{\circ}$ for different values of the radius $a$, as in Figure 7. A PEC target was used in the plot. As with dielectric targets, the internal reflections led to a less regular pattern. The results, calculated at three frequencies $(800 \mathrm{MHz}, 1 \mathrm{GHz}$, and $1.2 \mathrm{GHz}$ ), showed that different positions of the resonances were shifted coherently as the size of the wavelength was varied at a fixed cylinder's radius. 


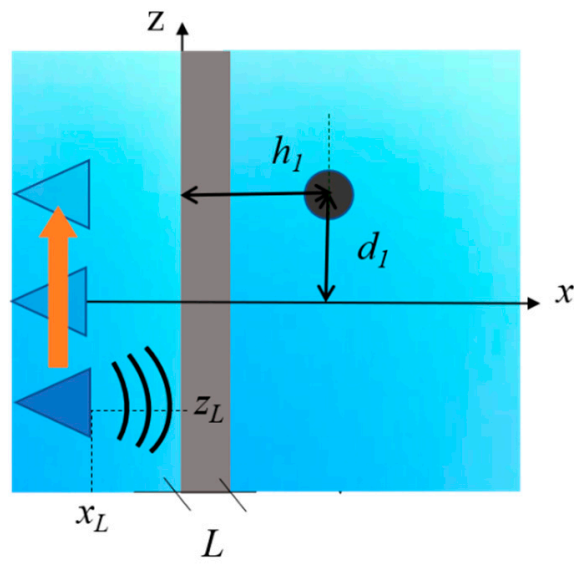

(a)

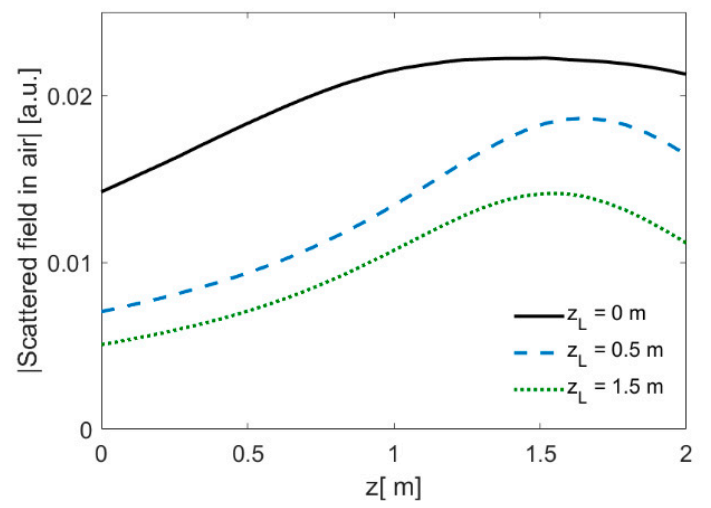

(b)

Figure 5. (a) Geometry of the problem for line-source scanning. (b) Scattered field in medium 0 along a line in $x=-50 \mathrm{~cm}$, under line source excitation, with a dielectric target $\left(\varepsilon_{\mathrm{rc}}=50\right)$ of radius $a=7.5 \mathrm{~cm}$ and center in $(0.6 \mathrm{~cm}, 0.5 \mathrm{~cm})$. The layer thickness is $L=20$ and relative permittivity $\varepsilon_{\mathrm{r} 1}=4$. The line source has its center in $\mathrm{x}_{\mathrm{L}}=-0.5 \mathrm{~m}$, and it is moved in different positions $\mathrm{z}_{\mathrm{L}}$.

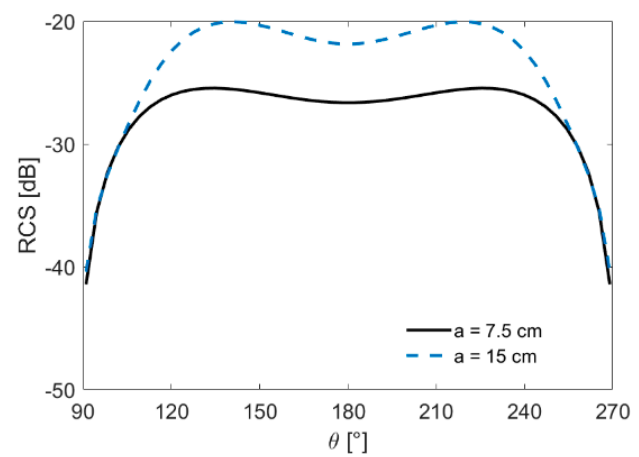

(a)

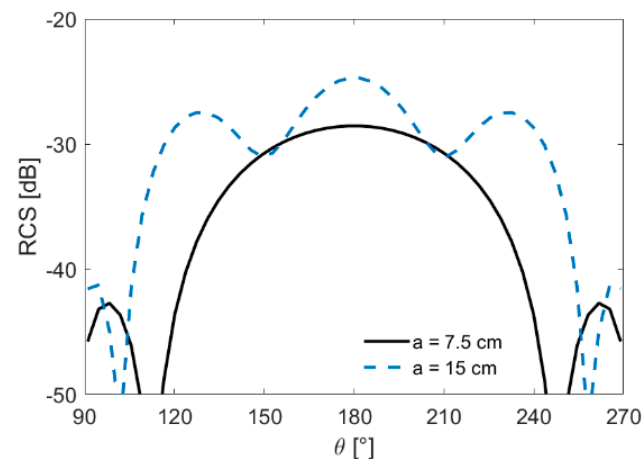

(b)

Figure 6. Radar cross sections as a function of the scattering angle $h$ at $1 \mathrm{GHz}$ : (a) $\varepsilon_{\mathrm{rc}}=50, \mathrm{~S}=1 \mathrm{~S} / \mathrm{m}$ (biological medium); and (b) $\varepsilon_{\mathrm{rc}}=2.5, \mathrm{~S}=0.005$ (wood).

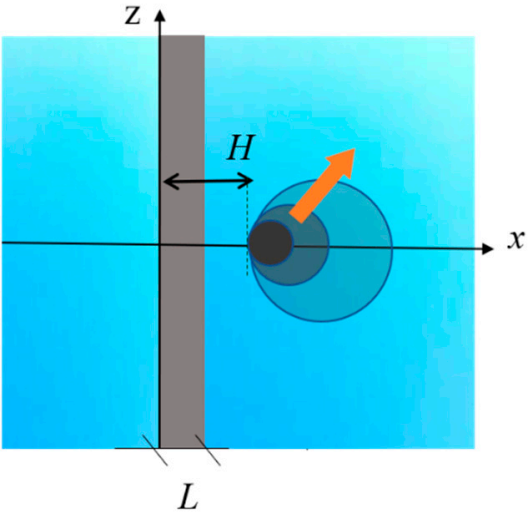

(a)

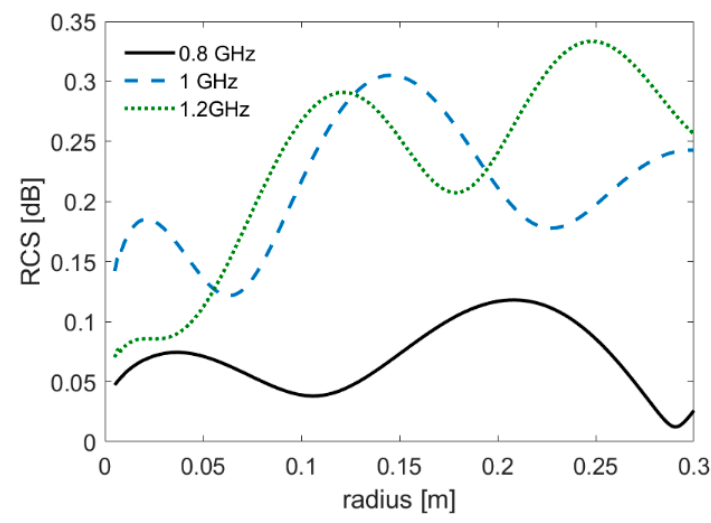

(b)

Figure 7. Radar cross section evaluated in the backscattering direction $\theta=180^{\circ}$, with $H=50 \mathrm{~cm}, \mathrm{PEC}$ target, $L=20 \mathrm{~cm}, \varepsilon_{\mathrm{r} 1}=4$ at three different frequencies.

\section{Conclusions}

The CWA implemented for the solution of scattering by targets in a through-wall environment has been reviewed in its non-iterative approach, where the multiple reflections relevant to the scattered 
field are expressed through a compact and convergent definition of the fields. The approach was partly extended to include the radiation from a line source as excitation field in the theoretical formulations. Targets may be either dielectric or PEC, and their size and permittivity can deal with targets of through-wall investigations. Numerical implementation is performed in an accurate way and through fast calculations, even for deeply buried targets. Results can be evaluated in both the near and far-field medium through the computations of the scattered field through all the media without asymptotic or approximated approaches. Following the approach developed in [18], the method may be extended to solve the time domain scattering from a pulsed source, and this would also allow for modeling of frequency-dispersive media. In the presented formulation, permittivity of the wall is purely real. A solution dealing with a lossy propagation medium may also be developed, employing inhomogeneous waves in the spectral integral relevant to the reflected and transmitted cylindrical waves, as in [26].

Author Contributions: Conceptualization, C.P. and G.S.; formal analysis, C.P. and G.S.; methodology, C.P. and G.S.; validation, C.P. and G.S.

Funding: This research was partly supported by the Italian Ministry for Education, University, and Research under the project PRIN2015 U-VIEW, grant number 20152HWRSL.

Conflicts of Interest: The authors declare no conflict of interest.

\section{References}

1. Amin, M.G. Through-the-Wall Radar Imaging; CRC Press: Boca Raton, FL, USA, 2010.

2. Peabody, J.E., Jr.; Charvat, G.L.; Goodwin, J.; Tobias, M. Through-the-Wall Imaging Radar. Linc. Lab. J. 2012, $19,62-72$.

3. Baranoski, E.J. Through wall imaging: Historical perspective and future directions. In Proceedings of the 2008 IEEE International Conference on Acoustics, Speech and Signal Processing (ICASSP), Las Vegas, NV, USA, 30 March-4 April 2008; pp. 5173-5176.

4. Soldovieri, F.; Solimene, R. Through-wall imaging via a linear inverse scattering algorithm. Geosci. Remote Sens. Lett. 2007, 4, 513-517. [CrossRef]

5. Giannopoulos, A. Modelling ground penetrating radar by GprMax. Constr. Build. Mater. 2005, 19, 755-762. [CrossRef]

6. Zhang, X.; Xi, X.; Li, M.; Wu, D. Comparison of Impulse Radar and Spread-Spectrum Radar in Through-Wall Imaging. IEEE Trans. Microw. Theory Tech. 2016, 64, 669-706. [CrossRef]

7. Dogaru, T. AFDTD User's Manual; ARL-TR-5145; Army Research Laboratory: Adelphi, MD, USA, 2010.

8. Wang, Y.; Safavi-Naeini, S.; Chaudhuri, S.K. A hybrid technique based on combining ray tracing and FDTD methods for site-specific modeling of indoor radio wave propagation. IEEE Trans. Antennas Propag. 2000, 48, 743-754. [CrossRef]

9. Reynaud, M.; Guiffaut, C.; Reineix, A.; Vauzolle, R. Modeling indoor propagation using an indirect hybrid method combining the UTD and the FDTD methods. In Proceedings of the 7th European Conference on Wireless Technology, Amsterdam, The Netherlands, 11-12 October 2004; pp. 345-348.

10. Gennarelli, G.; Vivone, G.; Braca, P.; Soldovieri, F.; Amin, M.G. Multiple Extended Target Tracking for Through-Wall Radars. IEEE Trans. Geosci. Remote Sens. 2015, 53, 6482-6494. [CrossRef]

11. Chang, P.C.; Burkholder, R.J.; Volakis, J.L.; Marhefka, R.J.; Bayram, Y. High-Frequency EM characterization of through-wall building imaging. IEEE Trans. Geosci. Remote Sens. 2009, 47, 1375-1387. [CrossRef]

12. Yektakhah, B.; Sarabandi, K. All-Directions Through-the-Wall Radar Imaging Using a Small Number of Moving Transceivers. IEEE Trans. Geosci. Remote Sens. 2016, 54, 6415-6428. [CrossRef]

13. di Vico, M.; Frezza, F.; Pajewski, L.; Schettini, G. Scattering by a finite set of perfectly conducting cylinders buried in a dielectric halfspace: A spectral-domain solution. IEEE Trans. Antennas Propag. 2005, 53, 719-727. [CrossRef]

14. di Vico, M.; Frezza, F.; Pajewski, L.; Schettini, G. Scattering by buried dielectric cylindrical structures. Radio Sci. 2005, 40, RS6S18. [CrossRef] 
15. Fiaz, M.A.; Frezza, F.; Pajewski, L.; Ponti, C.; Schettini, G. Scattering by a circular cylinder buried under a slightly rough surface: The cylindrical-wave approach. IEEE Trans. Antennas Propag. 2012, 60, $2834-2842$. [CrossRef]

16. Frezza, F.; Pajewski, L.; Ponti, C.; Schettini, G. Scattering by perfectly conducting circular cylinders buried in a dielectric slab through the cylindrical wave approach. IEEE Trans. Antennas Propag. 2009, 57, 1208-1217. [CrossRef]

17. Frezza, F.; Pajewski, L.; Ponti, C.; Schettini, G. Scattering by dielectric circular cylinders in a dielectric slab. J. Opt. Soc. Am. A 2010, 27, 687-695. [CrossRef] [PubMed]

18. Ponti, C.; Santariero, M.; Schettini, G. Electromagnetic scattering of a pulsed signal by conducting cylindrical targets embedded in a half-space medium. IEEE Trans. Antennas Propag. 2017, 65, 3073-3083. [CrossRef]

19. Frezza, F.; Pajewski, L.; Ponti, C.; Schettini, G. Through-wall electromagnetic scattering by $\mathrm{N}$ conducting cylinders. J. Opt. Soc. Am. A 2013, 30, 1632-1639. [CrossRef] [PubMed]

20. Vellucci, S.; Ponti, C. Scattering by a circular cylinder buried under a slightly rough surface: The cylindrical-wave approach. IEEE Trans. Microw. Theory Tech. 2015, 63, 30-39.

21. Orfanidis, S.J. Electromagnetic Waves and Antennas; Rutgers Univ.: Piscataway, NJ, USA, 2014; Available online: http://eceweb1.rutgers.edu/ \{\}orfanidi/ewa/ (accessed on 28 October 2019).

22. Balanis, C.A. Advanced Engineering Electromagnetics, 2nd ed.; John Wiley \& Sons: Hoboken, NJ, USA, 2012.

23. Elsherbeni, A.Z. A comparative study of two-dimensional multiple scattering techniques. Radio Sci. 1994, 29, 1023-1033. [CrossRef]

24. Estatico, C.; Fedeli, A.; Pastorino, M.; Randazzo, A. A Banach space regularization approach for multifrequency microwave imaging. Int. J. Antennas Propag. 2016, 2016, 9304371. [CrossRef]

25. Estatico, C.; Fedeli, A.; Pastorino, M.; Randazzo, A. A Banach space regularization approach for multifrequency microwave imaging. In Proceedings of the 13th European Conference on Antennas and Propagation (EUCAP), Krakow, Poland, 31 March-5 April 2019.

26. Frezza, F.; Pajewski, L.; Ponti, C.; Tedeschi, N.; Schettini, G. Electromagnetic Scattering by a Metallic Cylinder Buried in a Lossy Medium with the Cylindrical-Wave Approach. IEEE Geosci. Remote Sens. Lett. 2013, 10, 179-183. [CrossRef]

(C) 2019 by the authors. Licensee MDPI, Basel, Switzerland. This article is an open access article distributed under the terms and conditions of the Creative Commons Attribution (CC BY) license (http://creativecommons.org/licenses/by/4.0/). 